\title{
ARTí́CULO
}

\section{Variación espacio-temporal en la composición del ensamble de macroalgas del intermareal rocoso de Maitencillo, Valparaíso, costa central de Chile}

Spatio-temporal variation in the composition of the macroalgae assemblage of the intertidal rocky zone from Maitencillo, Valparaíso, central coast of Chile

\author{
Claudia Betancourtt ${ }^{1,2}$, Javier Zapata ${ }^{1,2}$, Nicolás Latorre ${ }^{1,2,3}$, Cristóbal Anguita ${ }^{1}$, \\ Francisco Castañeda ${ }^{1,2}$, Andrés Meynard ${ }^{1,2}$, Camila Fierro ${ }^{1}$, Camila Espinoza ${ }^{1,2}$, \\ Eduardo Guajardo ${ }^{1}$, Alejandra Núñez ${ }^{1,2}$, Nicolás Salas ${ }^{1,2}$, Constanza González, \\ María-Eliana Ramírez ${ }^{1,4}$, Cristian Bulboa-Contador ${ }^{1}$ y Loretto Contreras-Porcia ${ }^{1,2,5 *}$
}

${ }^{1}$ Departamento de Ecología y Biodiversidad, Facultad Ciencias de la Vida, Universidad Andres Bello, República 440, Santiago, Chile ${ }^{2}$ Centro de Investigación Marina Quintay (CIMARQ), Facultad Ciencias de la Vida, Universidad Andres Bello, Quintay, Chile ${ }^{3}$ Programa de Doctorado en Medicina de la Conservación, Facultad Ciencias de la Vida, Universidad Andres Bello, República 440, Santiago, Chile

${ }^{4}$ Museo Nacional de Historia Natural, Área Botánica, Casilla 787, Santiago, Chile

${ }^{5}$ Center of Applied Ecology \& Sustainability (CAPES-UC), Pontificia Universidad Católica de Chile, Avda. Libertador Bernardo O’Higgins 340, Santiago, Chile.*Autor de correspondencia: lorettocontreras@unab.cl

\begin{abstract}
Records on the diversity of algae serve as a foundation for establishing management and environmental protection programs, as well as for determining new commercial uses of algae. With this context in mind, the goal of the present study was to determine the richness, coverage, and composition of the macroalgae assemblage in the intertidal zone of Maitencillo, Valparaíso, Chile over the course of 3 years (2013-2015). A total of 29 species were recorded from 3 phyla - 23 Rhodophyta; 3 Ochrophyta, of the Phaeophyceae class; and 3 Chlorophyta. Species richness presented high spatial and temporal variations, with these variations most significantly explained by intertidal zone (61\%) and the seasons (31\%). The highest levels of specific richness were recorded in the mid and lower intertidal zones (14 species) in spring, as well as in the lower intertidal zone (14 species) during summer. The lowest values of specific richness were recorded for the mid intertidal zone ( 2 species) during fall. The distinct levels of the intertidal zone also notably impacted algae assemblage, explaining $53 \%$ of variation while the seasons explained $18 \%$ of variation. The greatest coverage was recorded in the mid intertidal zone during spring, with a predominance of Mazzaella laminarioides and Ulva spp. complex. In turn, the lowest coverage was found in fall, linked with processes of sand accretion. In rocky walls, the Pyropia complex dominated. Results indicate a marked pattern of vertical and seasonal distribution in the macroalgae assemblage, as well as notably high presence of Rhodophyta species. This benthic flora description serves to update information on the diversity of representative algal species from Valparaíso Region of Chile.
\end{abstract}

Key words: Seaweeds, intertidal distribution, coverage, richness

Resumen.- El registro de la diversidad de algas permite reconocer especies que podrían generar información base para medidas de manejo, protección de los ambientes y presentar nuevas oportunidades de uso. En este contexto, el objetivo del presente trabajo fue determinar la riqueza, cobertura y composición del ensamble de especies de macroalgas en la zona intermareal de Maitencillo, Valparaíso, Chile, durante 3 años de monitoreo (2013-2015). Un total de 29 especies fueron registradas, donde 23 de ellas pertenecen a Rhodophyta, 3 a Ochrophyta; clase Phaeophyceae, y 3 a Chlorophyta. La riqueza de especies presentó una alta variación espacio-temporal en el área de estudio, donde las zonas del intermareal y las estaciones del año presentaron un efecto significativo, explicando un 61 y $31 \%$ de su variabilidad, respectivamente. La mayor riqueza específica se registró en las zonas media y baja (14 especies) durante primavera, y en la zona baja del intermareal (14) durante verano. Los menores valores fueron encontrados en la zona media (2) durante otoño. Las zonas del intermareal demostraron ser la variable de mayor importancia a nivel de la comparación del ensamble de algas, explicando el $53 \%$ de la variación, mientras que la estacionalidad explicó el $18 \%$ de ésta. La mayor cobertura fue registrada en la zona media durante primavera, con predominio de Mazzaella laminarioides y Ulva spp. En la estación de otoño se obtuvo la menor cobertura debido a los procesos de acreción de arena. En paredones dominaron especies del complejo Pyropia. Los resultados indicaron un claro patrón de distribución vertical y variación estacional en el ensamble de macroalgas, destacando una mayor presencia de especies de Rhodophyta. La descripción de la flora bentónica obtenida en este trabajo actualiza la diversidad de algas representativa de la Región de Valparaíso, Chile.

Palabras clave: Macroalgas, distribución intermareal, cobertura, riqueza 


\section{INTRODUCCIÓN}

Las macroalgas desempeñan un importante rol en la producción primaria de los océanos, ya que sustentan las cadenas tróficas; siendo estructuradores comunitarios, otorgando sustrato, lugar de refugio y desove para invertebrados y peces (Cancino \& Santelices 1984, Vásquez 1992, Benavides et al. 1994, Ramírez 2006). En Chile, estudios que den cuenta de la diversidad de macroalgas en la zona central se remontan al realizado por Guiler (1959), seguido de Alveal (1970, 1971), Santelices \& Vera (1984), Hoffmann \& Santelices (1997), Ramírez \& Osorio (2000) y Ramírez et al. (2008); siendo el más actual Ramírez et al. (2018). Esto pone de manifiesto que aproximadamente cada 10 años, se realiza un estudio de la biota algal en zonas restringidas a lo largo de la costa chilena. En este sentido, hasta la década pasada, eran reconocidas al menos 444 especies de algas marinas para la costa de Chile (Ramírez 2006), número que ha ido en aumento con el desarrollo de estudios taxonómicos y moleculares (e.g., Contreras et al. 2007, Macaya \& Zuccarello 2010, Arakaki et al. 2011, González et al. 2012, Montecinos et al. 2012, Ramírez et al. 2014, Calderon \& Boo 2016, Guillemin et al. 2016), los que han demostrado la existencia de nuevos géneros y especies crípticas, con diversa disposición en la zona intermareal. En consecuencia, se hace necesario reevaluar constantemente la composición de la flora marina de Chile, aún más, si se considera la ocurrencia de cambios en los componentes bióticos de los ecosistemas marinos producto de la intervención del hombre, en donde una población importante de macroalgas se encuentra fuertemente presionadas por extracción y manejo con fines comerciales, evidenciando alteraciones en las poblaciones y comunidades afectadas (Vásquez et al. 2014). Además, el arribo y propagación de entidades algales a nuevas áreas del litoral chileno (Villaseñor-Parada et al. 2018) hace que los componentes florales deban ser observados frecuentemente para informar sobre posibles cambios en la distribución y composición en las comunidades costeras. Este es el caso de Codium fragile subsp. tomentosoides (van Goor) PC. Silva y su avance hacia el norte del país (Jofré et al. 2014); Schottera nicaeensis (JV. Lamouroux ex Duby) Guiry \& Hollenberg en la zona centro sur (Villaseñor et al. 2014) y Glaphyrosiphon chilensis ME Ramírez, Leister \& PW Gabrielson (Hommersand et al. 2010), con distribución en el sur del país, pero que también ha sido reportada de forma intermitente con masivas proliferaciones en localidades del litoral del centro de Chile (e.g., Grateloupia intestinalis (Harvey) Setchell ex PG. Parkinson, Collantes
\& Muñoz-Muga 2009). Por otro lado, en Chile, alrededor de 20 especies de algas marinas, muchas de ellas intermareales, tienen importancia comercial para la elaboración de diferentes productos demandados en mercados nacionales e internacionales, transformándose en un recurso económico que sustenta los sistemas de vida de comunidades costeras a lo largo de todo el litoral (Vásquez et al. 2014). En ese contexto, el registro de la biodiversidad de algas es, con seguridad, el primer paso para detectar especies con potencial económico e iniciar estrategias de explotación sustentable de estos recursos. El objetivo del presente trabajo fue determinar la riqueza, cobertura y composición del ensamble de macroalgas durante los años 2013-2015, en la zona central de Chile, particularmente en la localidad de Maitencillo (32 $29^{\circ} \mathrm{S}$; $71^{\circ} 26^{\prime} \mathrm{O}$ ), situada $11 \mathrm{~km}$ al norte de la comuna de Puchuncaví, en la Región de Valparaíso, Chile; zona costera que ha sido foco de estudios de diferentes trabajos científicos en algas (e.g., Contreras et al. 2005, González et al. 2012, 2014; Montecinos et al. 2012, Flores-Molina et al. 2014, Ramírez et al. 2014, Sordet et al. 2014, González \& Santelices 2017).

\section{Materiales y MÉtodos}

El estudio fue realizado en la zona intermareal de la localidad de Maitencillo ( $31^{\circ} 29^{\prime} \mathrm{S}$; $71^{\circ} 26^{\prime} \mathrm{O}$ ), Chile central, Región de Valparaíso; específicamente en la localidad de Aguas Blancas, a lo largo de 90-100 m de línea de costa incluyendo las zonas altas, medias, bajas y paredones (Fig. 1). Se efectuó un muestreo mensual determinando la riqueza y cobertura de las especies de algas durante el 2013, 2014 y 2015. Para esto, se usaron 2 transectos perpendiculares a la línea de marea, distanciados 30-35 m uno del otro. Ambos transectos se emplearon para obtener una mayor información sobre las especies presentes y determinar los patrones estacionales de cobertura de cada uno de ellos. En cada transecto, las especies fueron identificadas desde la zona alta del intermareal hasta la zona baja, donde se encuentra el cinturón de Lessonia spicata (Suhr) Santelices. No se registraron pozas intermareales a lo largo de los transectos verticales. Se incluyeron en los análisis los paredones, ya que la intensidad de los factores físicos en esta zona es diferencial con respecto a la zona alta (Zapata 2016). Para la determinación de la cobertura, se utilizó el método de intersección de puntos sobre cuadrantes reticulados de $0,25 \mathrm{~m}^{2}$. En cada zona intermareal, se utilizó un número distinto de cuadrantes de acuerdo con la superficie de estos; en la zona alta entre 7 a 11, en la zona media entre 11 y 30 cuadrantes, en la zona baja entre 5 a 10, y en el 


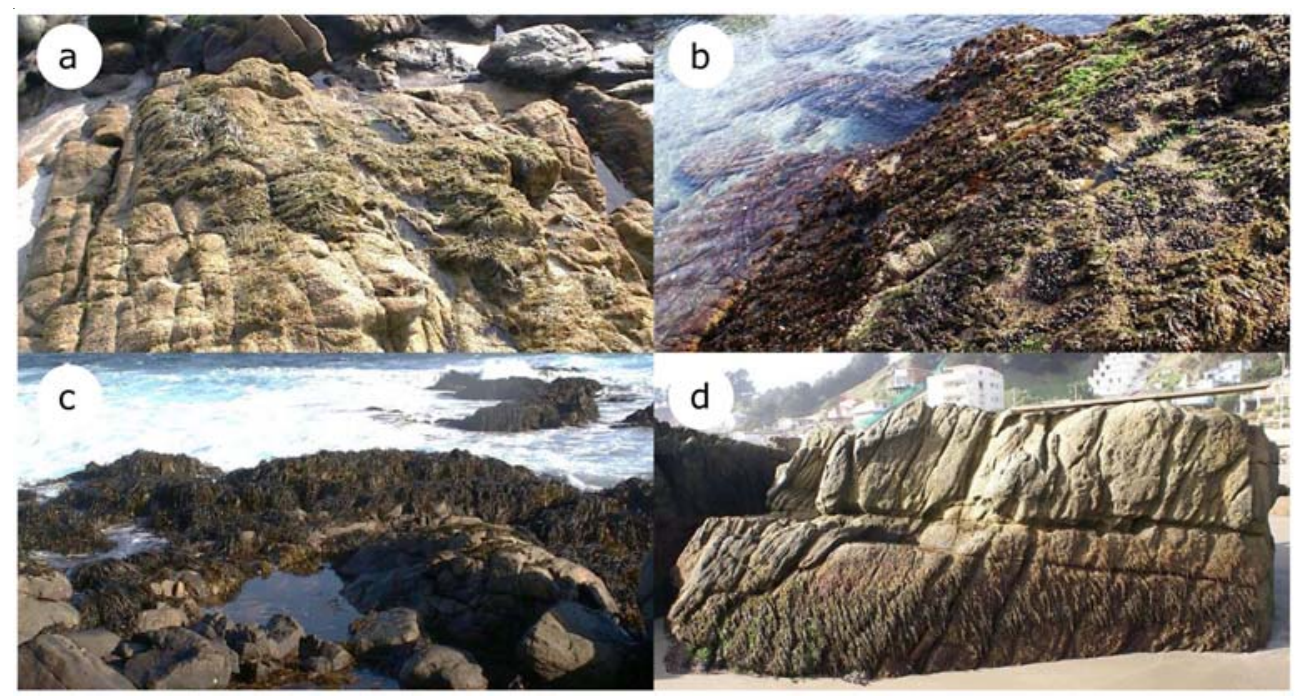

Figura 1. Biotopos caracterizados en la zona intermareal de Maitencillo, Valparaíso: a) intermareal alto, b) medio, (c) bajo, (d) paredón / Characterized biotopes of the intertidal zone of Maitencillo, Valparaíso: a) high intertidal, b) medium intertidal, c) low intertidal, d) steep wall

paredón se utilizaron 3 cuadrantes. La determinación de la cobertura (\%) total por especie fue obtenida promediando los resultados entre los cuadrantes para cada zona. Para la correcta identificación de las especies, se recolectaron individuos completos, los cuales fueron transportados a la unidad 'Laboratorio de Ecología y Biología Molecular en Macroalgas (LEBMA)’, Departamento de Ecología y Biodiversidad de la Universidad Andrés Bello. Parte del material recolectado fue herborizado y otra parte de la muestra fue conservada en sílica gel (Vetec Analytical Reagents, Brasil) para estudios moleculares. La identificación taxonómica se realizó utilizando los métodos tradicionales para este tipo de estudio morfológico (Womersley 1984), basándose en la literatura más actualizada depositada y validada por Guiry \& Guiry (2018). Los cortes histológicos del material fresco de los especímenes colectados fueron observados a través de un microscopio de luz (Leica ${ }^{\circledR}$, Microsystems, modelo DM 500, Suiza), acoplado a un sistema de imagen HD, conectado al software LAS EZ (Leica Microsystems).

\section{Análisis de DATOS}

\section{Riqueza DE ESPECIES}

Para determinar la variación espacial (zonas del intermareal) y estacional (verano, otoño, invierno y primavera) de la riqueza de especies, se realizó un modelo lineal generalizado con una distribución de Poisson (Zuur et al. 2009). Se modeló la riqueza total obtenida de un total de 135 cuadrantes analizados durante el periodo de estudio en respuesta a las zonas del intermareal (como variable categórica), las estaciones (como variable categórica) y a la interacción de ambas variables. El modelo fue seguido por un análisis de devianza (equivalente a un 'ANDEVA'). Estos análisis se realizaron utilizando el paquete 'gamlss' (Rigby \& Stasinopoulos 2005) de R (R Core Team 2017).

\section{COBERTURA Y COMPOSICIÓN DEL ENSAMBLE DE ESPECIES}

Para describir los patrones de cobertura y composición del ensamble de especies de macroalgas, obtenidos de un total de 135 cuadrantes analizados durante el periodo de estudio, se utilizó el porcentaje de cobertura (\%). Se realizó un modelo lineal generalizado multivariado (multiGLM) con una distribución binomial negativa (apropiada para casos de sobre dispersión, Zuur et al. 2009, O’hara \& Kotze 2010). Se consideró a todas las especies (i.e., 14) que presentaron una probabilidad de ocurrencia $>5 \%$ durante el periodo de estudio. Los multiGLM son una aproximación analítica relativamente nueva, que ha demostrado ser más robusta en relación a los análisis tradicionales basados en distancia, los cuales suelen confundir los efectos de locación con aquellos de dispersión (Warton et al. 2012). Se modeló la composición del ensamble de macroalgas en respuesta a las zonas del intermareal, a las estaciones y a la interacción de ambas variables. El modelo fue seguido de un análisis de devianza utilizado el remuestreo 'pit.trap' con 1000 iteraciones. Adicionalmente, se realizaron modelos univariados para todas las especies, con 
las mismas especificaciones descritas anteriormente. Estos análisis se realizaron usando el paquete 'mvabund' (Wang et al. 2012) de R (R Core Team 2017).

\section{Resultados}

\section{VARIACIÓN ESPACIO-TEMPORAL DE LA RIQUEZA DE MACROALGAS}

Un total de 29 especies fueron registradas en las 4 zonas del intermareal (alto, medio, bajo y de paredones), a lo largo del periodo de estudio. 23 de ellas fueron adscritas a la división Rhodophyta, 3 a Ochrophyta; clase Phaeophyceae y 3 a Chlorophyta (Tabla 1, Fig. 2). La riqueza de especies presentó una alta variación espaciotemporal en el área de estudio (Tabla 2, Fig. 3). La mayor riqueza fue encontrada para las zonas media (14 especies) y baja (14) durante primavera; y en la zona baja del intermareal (14) durante verano. Durante el otoño, los menores valores fueron encontrados en la zona media (2 especies), y nula presencia de especies en la zona alta y de paredones (Tablas 1, 2 y 3). En la zona de paredones la máxima riqueza (4 especies) fue registrada durante primavera. La mayor riqueza total hallada durante el periodo de estudio fue de 22 especies durante primavera y la menor fue de solo 6 especies durante el otoño (Tablas 1, 2 y 3). Finalmente, las zonas del intermareal y las estaciones del año presentaron un efecto significativo ( $P$ $<0,05$, Tabla 3) en la riqueza de especies, explicando un 61 y un 31\% de su variabilidad, respectivamente. El efecto interactivo de ambas variables predictivas explicó un 79\% de la variabilidad de la riqueza.

Tabla 1. Lista de las especies encontradas a nivel estacional en Maitencillo, Valparaíso. X indica presencia de la especie. I: invierno, P: primavera, V: verano; O: otoño. *de acuerdo a Guillemin et al. (2016) e identificados molecularmente (Meynard in prep). Ver Figura 2 para los hábitos de la mayor parte de las especies registradas / List of species found in different zones (1 and 2) and seasons at Maitencillo, Valparaíso. $\mathrm{X}$ indicates species presence. I: winter, P: spring, V: summer; O: fall. *according to Guillemin et al. (2016) and molecularly identified (Meynard in prep). See Figure 2 for the habits of most registered species

\begin{tabular}{|c|c|c|c|c|}
\hline \multirow{2}{*}{ Especies } & \multicolumn{4}{|c|}{ Estaciones } \\
\hline & I & $\mathrm{P}$ & $\mathrm{V}$ & $\mathrm{O}$ \\
\hline \multicolumn{5}{|l|}{ Rhodophyta } \\
\hline Ahnfeltiopsis furcellata (C.Agardh) P.C.Silva \& DeCew & & $\mathrm{x}$ & $\mathrm{x}$ & \\
\hline Centroceras clavulatum (C. Agardh) Montagne & & & $\mathrm{x}$ & \\
\hline Ceramium sp. & & $\mathrm{x}$ & $\mathrm{x}$ & \\
\hline Chondria secundata (J. Agardh) De Toni & & $\mathrm{x}$ & $\mathrm{x}$ & \\
\hline Chondrus canaliculatus (C. Agardh) Greville & & $\mathrm{x}$ & $\mathrm{x}$ & \\
\hline Corallina officinalis Linnaeus & $\mathrm{x}$ & $\mathrm{x}$ & $\mathrm{x}$ & \\
\hline Gastroclonium cylindricum Santelices, I.A.Abbott \& Ramírez & & $\mathrm{x}$ & $\mathrm{x}$ & $\mathrm{x}$ \\
\hline Gelidium chilense (Montagne) Santelices \& Montalva & & $\mathrm{x}$ & $\mathrm{x}$ & \\
\hline Gelidium lingulatum Kützing & $\mathrm{x}$ & $\mathrm{x}$ & $\mathrm{x}$ & $\mathrm{x}$ \\
\hline Gelidium pusillum (Stackhouse) Le Jolis & $\mathrm{x}$ & & & \\
\hline Grateloupia doryphora (Montagne) M.Howe & & $\mathrm{x}$ & & \\
\hline Gymnogongrus sp. & $\mathrm{x}$ & & & \\
\hline Lithothamnion sp. & $\mathrm{x}$ & $\mathrm{x}$ & & \\
\hline Mazzaella laminarioides (Bory de Saint-Vincent) Fredericq & $\mathrm{x}$ & $\mathrm{x}$ & $\mathrm{x}$ & $\mathrm{x}$ \\
\hline Montemaria horridula (Montagne) A.B. Joly \& Alveal & $x$ & & & \\
\hline Nothogenia fastigiata (Bory) P.G. Parkinson & $\mathrm{x}$ & $\mathrm{x}$ & $\mathrm{x}$ & \\
\hline Petroglossum pacificum Hollenberg & $\mathrm{x}$ & & & \\
\hline Polysiphonia sp. & & & & $\mathrm{x}$ \\
\hline *Porphyra sp. CHE & & $\mathrm{x}$ & & \\
\hline *Porphyra sp. CHC & $\mathrm{x}$ & & & \\
\hline *Pyropia sp. CHK & $\mathrm{x}$ & $\mathrm{x}$ & $\mathrm{x}$ & \\
\hline Pyropia orbicularis ME. Ramírez, L. Contreras-Porcia \& ML. Guillemin & $\mathrm{x}$ & $\mathrm{x}$ & $\mathrm{x}$ & \\
\hline Trematocarpus dichotomus Kützing & & $\mathrm{x}$ & $\mathrm{x}$ & \\
\hline \multicolumn{5}{|l|}{ Chlorophyta } \\
\hline Chaetomorpha firma Levring & $\mathrm{x}$ & $\mathrm{x}$ & & \\
\hline Codium bernabei A.V. González, M.E.Chacana \& P.C.Silva & $\mathrm{x}$ & $\mathrm{x}$ & $\mathrm{x}$ & $\mathrm{x}$ \\
\hline Ulva spp. & $\mathrm{x}$ & $\mathrm{x}$ & $\mathrm{x}$ & $\mathrm{x}$ \\
\hline \multicolumn{5}{|l|}{ Ochrophyta } \\
\hline Dictyota kunthii (C.Agardh) Greville & & $\mathrm{x}$ & $\mathrm{x}$ & \\
\hline Myriogloea chilensis (Montagne) A.H.Llaña & & $\mathrm{x}$ & & \\
\hline Scytosiphon gracilis Kogame & & $\mathrm{x}$ & & \\
\hline
\end{tabular}



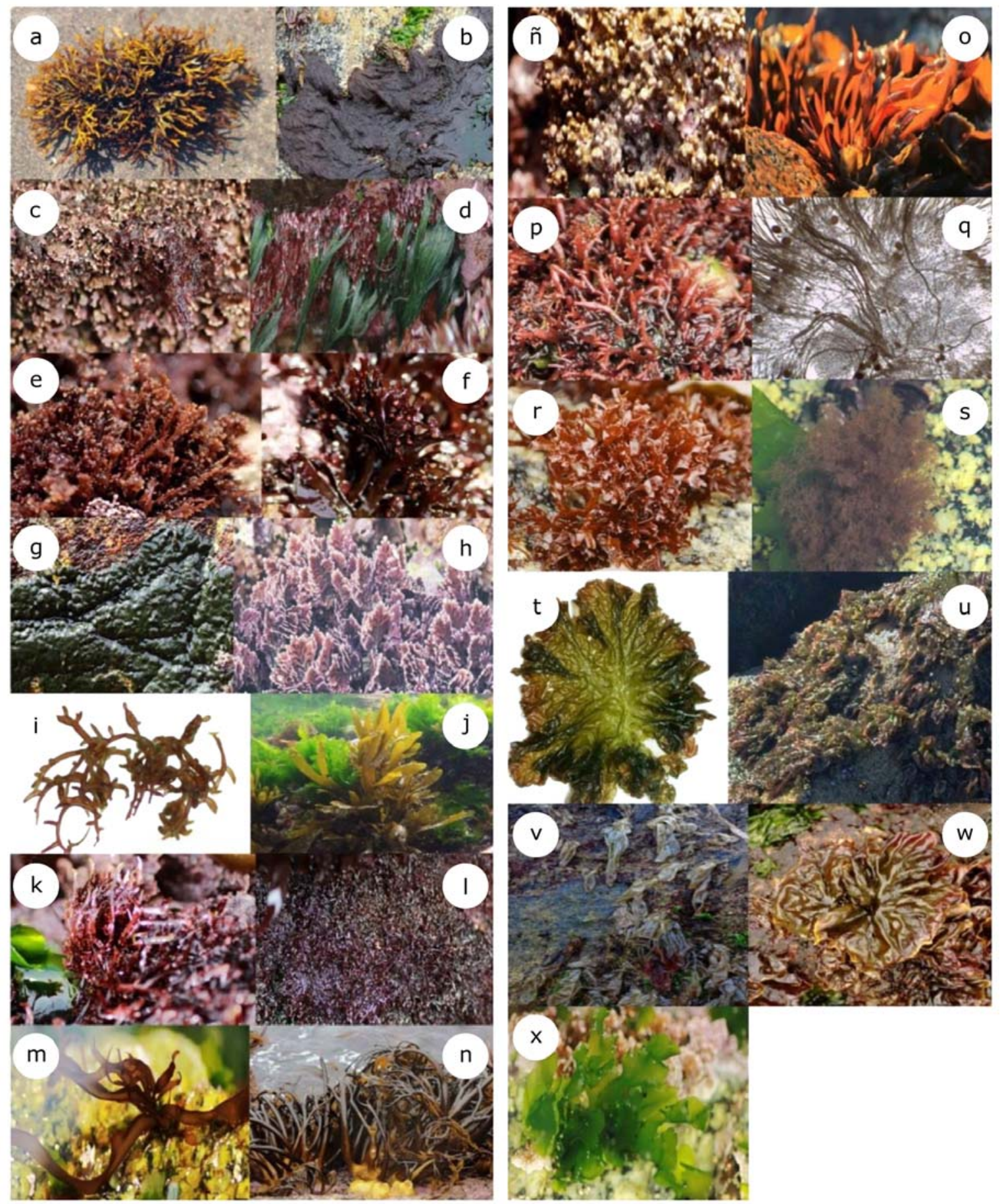

Figura 2. Especies presentes en el intermareal de Maitencillo, Valparaíso. (a) Ahnfeltiopsis furcellata, (b) Centroceras clavulatum, (c) Ceramium sp., (d), Chaetomorpha firma, (e) Chondria secundata, (f) Chondrus canaliculatus, (g) Codium bernabei, (h) Corallina officinalis, (i) Gastroclonium cylindricum, (j) Dictyota kunthii, (k) Gelidium lingulatum, (I), Gelidium chilense, (m) Grateloupia doryphora, (n) Lessonia spicata, (ñ) Lithothamnion sp., (o) Mazzaella laminarioides, (p) Montemaria horridula, (q) Myriogloea chilensis, (r) Nothogenia fastigiata, (s) Polysiphonia sp., (t) Porphyra sp. CHE, (u) Porphyra sp. CHC, (v) Pyropia sp. CHK, (w) Pyropia orbicularis y (x) Ulva spp. / Species present at the intertidal of Maitencillo, Valparaíso 
Tabla 2. Riqueza de especies por zona intermareal y estaciones del año en Maitencillo, Valparaíso / Species richness by intertidal zone and season at Maitencillo, Valparaíso

\begin{tabular}{|c|c|c|c|c|}
\hline $\begin{array}{c}\text { Nivel } \\
\text { intermareal }\end{array}$ & Primavera & Verano & Otoño & Invierno \\
\hline Alto & $\begin{array}{l}\text { Chaetomorpha firma } \\
\text { Mazzaella laminarioides } \\
\text { Porphyra sp. CHE } \\
\text { Pyropia orbicularis } \\
\text { Pyropia sp. CHK } \\
\text { Ulva spp. }\end{array}$ & $\begin{array}{l}\text { Mazzaella laminarioides } \\
\text { Pyropia orbicularis } \\
\text { Pyropia sp. CHK } \\
\text { Ulva spp. }\end{array}$ & & $\begin{array}{l}\text { Porphyra sp. CHC } \\
\text { Pyropia orbicularis } \\
\text { Pyropia sp. CHK } \\
\text { Ulva spp. }\end{array}$ \\
\hline Medio & $\begin{array}{l}\text { Ahnfeltiopsis furcellata } \\
\text { Ceramium sp. } \\
\text { Chondria secundata } \\
\text { Chondrus canaliculatus } \\
\text { Codium bernabei } \\
\text { Gelidium chilense } \\
\text { Gelidium lingulatum } \\
\text { Grateloupia doryphora } \\
\text { Lithothamnion sp. } \\
\text { Mazzaella laminarioides } \\
\text { Nothogenia fastigiata } \\
\text { Pyropia orbicularis } \\
\text { Pyropia sp. CHK } \\
\text { Ulva spp. }\end{array}$ & $\begin{array}{l}\text { Chondrus canaliculatus } \\
\text { Codium bernabei } \\
\text { Gelidium chilense } \\
\text { Gelidium lingulatum } \\
\text { Mazzaella laminarioides } \\
\text { Nothogenia fastigiata } \\
\text { Pyropia orbicularis } \\
\text { Pyropia } \text { sp. CHK } \\
\text { Trematocarpus dichotomus } \\
\text { Ulva spp. }\end{array}$ & $\begin{array}{l}\text { Gelidium lingulatum } \\
\text { Mazzaella laminarioides }\end{array}$ & $\begin{array}{l}\text { Chaetomorpha firma } \\
\text { Gelidium lingulatum } \\
\text { Gelidium pusillum } \\
\text { Mazzaella laminarioides } \\
\text { Montemaria horridula } \\
\text { Nothogenia fastigiata } \\
\text { Pyropia orbicularis } \\
\text { Pyropia sp. CHK } \\
\text { Ulva spp. }\end{array}$ \\
\hline Bajo & $\begin{array}{l}\text { Ahnfeltiopsis furcellata } \\
\text { Ceramium sp. } \\
\text { Chondria secundata } \\
\text { Chondrus canaliculatus } \\
\text { Codium bernabei } \\
\text { Corallina officinalis } \\
\text { Dictyota kunthii } \\
\text { Gastroclonium cylindricum } \\
\text { Gelidium lingulatum } \\
\text { Mazzaella laminarioides } \\
\text { Myriogloea chilensis } \\
\text { Nothogenia fastigiata } \\
\text { Trematocarpus dichotomus } \\
\text { Ulva spp. }\end{array}$ & $\begin{array}{l}\text { Ahnfeltiopsis furcellata } \\
\text { Centroceras clavulatum } \\
\text { Ceramium sp. } \\
\text { Chondria secundata } \\
\text { Chondrus canaliculatus } \\
\text { Codium bernabei } \\
\text { Corallina officinalis } \\
\text { Dictyota kunthii } \\
\text { Gastroclonium } \\
\text { cylindricum. } \\
\text { Gelidium chilense } \\
\text { Mazzaella laminarioides } \\
\text { Nothogenia fastigiata } \\
\text { Trematocarpus dichotomus } \\
\text { Ulva spp. }\end{array}$ & $\begin{array}{l}\text { Gelidium lingulatum } \\
\text { Codium bernabei } \\
\text { Gastroclonium cylindricum } \\
\text { Mazzaella laminarioides } \\
\text { Polysiphonia sp. } \\
\text { Ulva } \text { spp. }\end{array}$ & $\begin{array}{l}\text { Ceramium sp. } \\
\text { Chaetomorpha firma } \\
\text { Codium bernabei } \\
\text { Corallina officinalis } \\
\text { Dictyota kunthii } \\
\text { Gelidium lingulatum } \\
\text { Gymnogongrus sp. } \\
\text { Lithothamnion sp. } \\
\text { Mazzaella laminarioides } \\
\text { Nothogenia fastigiata } \\
\text { Petroglossum pacificum } \\
\text { Ulva } \text { spp. }\end{array}$ \\
\hline Paredón & $\begin{array}{l}\text { Pyropia orbicularis } \\
\text { Pyropia } \mathrm{sp} . \mathrm{CHK} \\
\text { Scytosiphon gracilis } \\
\text { Ulva spp. }\end{array}$ & Pyropia sp. CHK & & Pyropia sp. CHK \\
\hline
\end{tabular}

Tabla 3. Resultado del modelo lineal generalizado para la variación espacio-temporal de la riqueza de especies de macroalgas en el intermareal rocoso de Maitencillo, Valparaíso, Chile. Se muestra la riqueza de especies en respuesta a las zonas del intermareal (alto, medio, bajo y paredón), las estaciones (primavera, verano, otoño e invierno) y la interacción de ambas variables. $R^{2}$ indica el \% de variación explicada. GL; grados de libertad, TRL; test de razón de máxima verosimilitud, $\operatorname{Pr}(\mathrm{Chi})$ : valor $\boldsymbol{P} /$ Result of generalized linear model for the spatial-temporal variation of the macroalgae species richness at the rocky intertidal of Maitencillo, Valparaíso, Chile. The species richness is shown in response to intertidal zones (high, medium, low and wall), seasons (spring, summer, autumn and winter) and interaction of both variables. $R^{2}$ indicates the \% of explained variation. GL; degrees of freedom, TRL; Likelihood ratio test, $\operatorname{Pr}(\mathrm{Chi}) ; P$-values

\begin{tabular}{lcccc}
\hline & GL & TRL & $\operatorname{Pr}($ Chi) & $\mathrm{R}^{2}$ \\
\hline Zona & 3 & 122,94 & $<0,0001$ & 0,61 \\
Estación & 3 & 46,91 & $<0,0001$ & 0,32 \\
Zona x Estación & 9 & 38,074 & $<0,0001$ & 0,79 \\
\hline
\end{tabular}

Entre las especies de Maitencillo destacan Mazzaella laminarioides (Bory de Saint-Vincent) Fredericq (Rhodophyta, Gigartinales), aquellas que forman el complejo Ulva spp., Gelidium lingulatum Kützing (Rhodophyta, Gelidiales) y Codium bernabei A.V. González, M.E. Chacana \& P.C. Silva (Chlorophyta, Bryopsidales), las cuales fueron registradas en todas las estaciones evaluadas (Tabla 1). Nothogenia fastigiata (Bory) P.G. Parkinson (Rhodophyta, Nemaliales), Pyropia sp. CHK (Guillemin et al. 2016) y Pyropia orbicularis ME. Ramírez, L. Contreras-Porcia \& ML. Guillemin (Rhodophyta, Bangiales) fueron registradas predominantemente en invierno, primavera y verano (Tabla 1). 


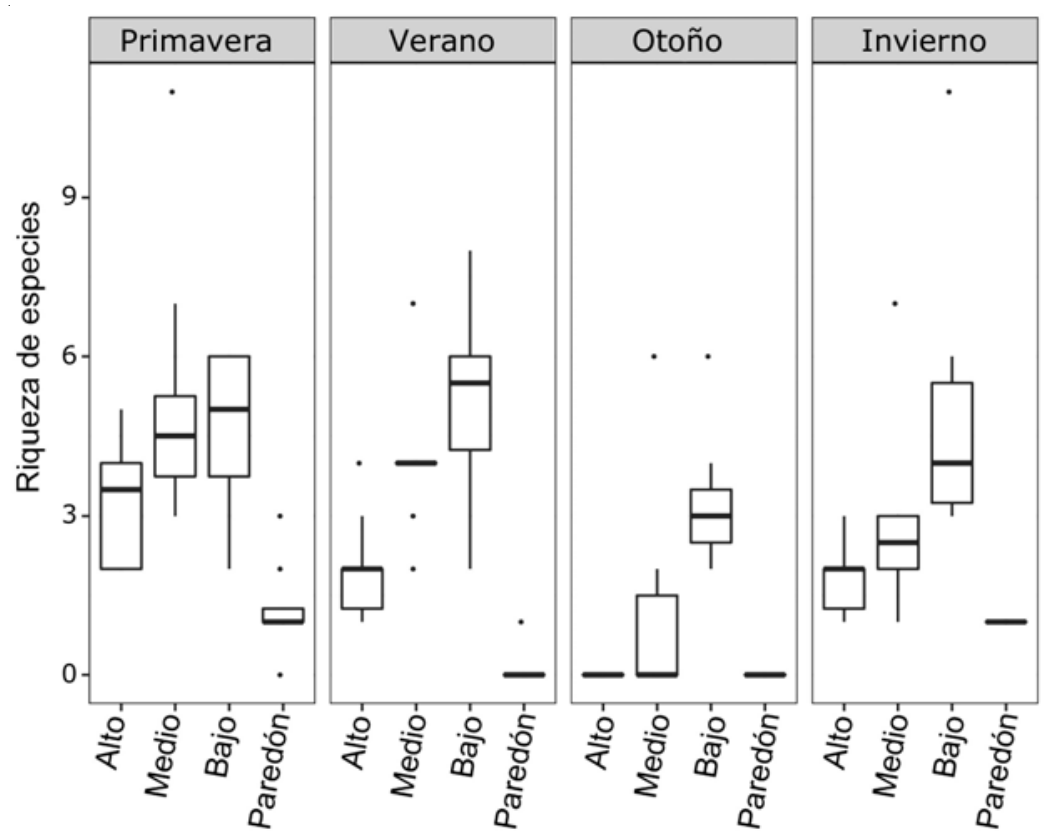

Figura 3. Gráfico de cajas de la variación espacio-temporal de la riqueza de especies macroalgales en la zona intermareal de Maitencillo, Valparaíso / Boxplot of spatio-temporal variation of the richness of macroalgal species at the intertidal zone of Maitencillo, Valparaíso

\section{VARIACIÓN ESPACIO-TEMPORAL EN LA COBERTURA Y COMPOSICIÓN DE MACROALGAS EN EL INTERMAREAL}

La composición del ensamble de macroalgas (14 especies analizadas) presentó una variación espacio-temporal significativa (multiGLM, $P<0,05$, Tabla 4). Las zonas del intermareal mostraron ser la variable de mayor importancia, explicando el 53\% de la variación del ensamble de macroalgas, mientras que la estacionalidad explicó el 18\% de su variabilidad. La interacción de ambas variables presentó un efecto significativo en la composición, explicando el 29\% de su variabilidad. El 93 y 43\% de las especies analizadas presentó una variación espacial y estacional, respectivamente, significativa a nivel de la cobertura ( $P<0,05$, Tabla 5). Finalmente, para el 50\% de las especies se evidenció un efecto significativo en la interacción de ambas variables.

\section{ESPECIES REPRESENTATIVAS POR ZONA INTERMAREAL}

Zona intermareal alta (Fig. 4a): Pyropia orbicularis presentó una marcada estacionalidad, con una mayor cobertura en primavera (12 $\pm 2 \%$ ) y verano $(7,4 \pm 1,5 \%)$. Pyropia sp. CHK mostró una alta cobertura durante primavera (21 $\pm 1,2 \%$ ) y durante invierno (19,6 $\pm 3,7 \%)$, al
Tabla 4. Resultado del modelo lineal generalizado multivariado para la variación espacio-temporal de la composición de la cobertura de macroalgas en el intermareal rocoso de Maitencillo, Valparaíso, Chile. Se muestran los resultados para la composición de especies en respuesta a las zonas del intermareal (alto, medio, bajo y paredón), las estaciones (primavera, verano, otoño e invierno) y la interacción de ambas variables. Ver Tabla 5 para los resultados de los modelos univariados por especies. Res.GL; grados de libertad de los residuales, Dev; desviación, $\operatorname{Pr}(<\mathrm{Dev})$; valor $\boldsymbol{P}$ / Result of multivariate generalized linear model for the spatiotemporal variation of the macroalgae cover composition at the rocky intertidal of Maitencillo, Valparaíso, Chile. The results are shown for the composition of species in response to intertidal zones (high, medium, low and wall), seasons (spring, summer, autumn and winter) and interaction of both variables. See Table 5 for the results of the univariate models by species. Res.GL; Residual degrees of freedom, Dev; deviance, $\operatorname{Pr}(>\operatorname{Dev}) ; P$-values

\begin{tabular}{lcccc}
\hline & Res.GL & GL & Dev & $\operatorname{Pr}(>$ Dev $)$ \\
\hline Zona & 131 & 3 & 385,5 & 0,001 \\
Estación & 128 & 3 & 129,1 & 0,001 \\
Zona x Estación & 119 & 9 & 207,9 & 0,001 \\
\hline
\end{tabular}


Tabla 5. Resultados de modelos lineales generalizado para la variación espacio-temporal de la cobertura de las principales especies de macroalgas en el intermareal rocoso de Maitencillo, Valparaíso. Las especies se encuentran ordenadas de mayor a menor devianza (Dev) explicada sobre la variación espacial y temporal del ensamble de macroalgas. Ver Tabla 4 para resultado del modelo multivariado / Results of generalized linear models for the spatio-temporal variation of the coverage of the main macroalgal species at the rocky intertidal of Maitencillo, Valparaíso. Species are ordered from highest to lowest deviance (Dev), explained on the spatial and temporal variation of the macroalgal assemblage. See Table 4 for the result of the multivariate model. Per(Dev); P-values

\begin{tabular}{lcccccc}
\hline \multirow{2}{*}{ Especie } & \multicolumn{2}{c}{ Zona } & \multicolumn{2}{c}{ Estación } & \multicolumn{2}{c}{ Zona x Estación } \\
\cline { 2 - 7 } & Dev & $\operatorname{Pr}(>\operatorname{Dev})$ & $\operatorname{Dev}$ & $\operatorname{Pr}(>\operatorname{Dev})$ & $\operatorname{Dev}$ & $\operatorname{Pr}(>\operatorname{Dev})$ \\
\hline Porphyra sp. CHC & 48,203 & 0,001 & 10,871 & 0,022 & 18,315 & 0,006 \\
Codium bernabei & 46,368 & 0,001 & 3,882 & 0,361 & 12,287 & 0,017 \\
Pyropia sp. CHK & 39,912 & 0,001 & 23,46 & 0,001 & 11,421 & 0,002 \\
Ceramium sp. & 36,849 & 0,001 & 14,789 & 0,003 & 0,001 & 0,401 \\
Chondrus canaliculatus & 33,032 & 0,001 & 9,031 & 0,054 & 0,001 & 0,389 \\
Gelidium chilense & 31,683 & 0,001 & 1,288 & 0,833 & 3,622 & 0,185 \\
Pyropia orbicularis & 30,782 & 0,001 & 18,844 & 0,002 & 29,964 & 0,001 \\
Mazzaella laminarioides & 28,064 & 0,001 & 1,458 & 0,557 & 51,858 & 0,001 \\
Dictyota kunthii & 26,575 & 0,001 & 31,407 & 0,001 & 0,002 & 0,378 \\
Ulva spp. & 18,891 & 0,001 & 4,762 & 0,261 & 45,023 & 0,001 \\
Trematocarpus dichotomus & 16,627 & 0,001 & 1,624 & 0,755 & 6,445 & 0,042 \\
Ahnfeltiopsis furcellata & 12,7 & 0,008 & 1,876 & 0,713 & 3,785 & 0,166 \\
Nothogenia fastigiata & 9,261 & 0,02 & 3,411 & 0,429 & 16,155 & 0,123 \\
Gelidium lingulatum & 6,591 & 0,105 & 2,445 & 0,62 & 9,028 & 0,141 \\
\hline
\end{tabular}

igual que Porphyra sp. CHC en esta última estación (4,7 $\pm 1,6 \%)$. Finalmente, el complejo Ulva spp. exhibió la mayor cobertura en primavera (6-9\%).

Zona intermareal media (Fig. 4b): Mazzaella laminarioides fue predominante en esta zona con un 35$33 \%$ de cobertura durante primavera y verano, seguido del complejo Ulva spp. (14\%) y Nothogenia fastigiata, con un menor porcentaje de cobertura (7-4\%). Durante el otoño, Mazzaella laminarioides y Gelidium lingulatum alcanzaron una baja cobertura ( 0,8 y $0,6 \%$, respectivamente).

Zona intermareal baja (Fig. 5a): La mayor cobertura de especies fue determinada en esta zona en todas las estaciones. El complejo Ulva spp. (6-27\%) y Mazzaella laminarioides (11-20\%) fueron predominantes, con las mayores coberturas. Myriogloea chilensis (Montagne) A.H.Llaña presentó una marcada estacionalidad, estando presente solo en primavera con un 2,2 $\pm 1,1 \%$ de cobertura, de igual manera Gelidium chilense (Montagne) Santelices \& Montalva en la época de verano con un 1,6 \pm 0,9\%.

Zona de paredones (Fig. 5b): Se registró una alta cobertura de Pyropia sp. CHK (38\% primavera-55\% invierno). Pyropia orbicularis presentó una baja cobertura $(1,4 \pm 0,9 \%)$ y solo estuvo presente durante primavera. El complejo Ulva spp. alcanzó durante primavera un $2,6 \pm 0,98 \%$ de cobertura.

\section{Discusión}

Los resultados obtenidos en este estudio descriptivo indican que existen importantes diferencias en la riqueza, cobertura y composición de especies a lo largo de la zona intermareal como también a nivel estacional; las cuales han sido demostradas en trabajos ecológicos previos en otras áreas costeras (Zardi et al. 2013, Raffo et al. 2014, Zapata 2016). Particularmente, la riqueza de especies presentó una alta variación espacio-temporal en el área de estudio, donde las zonas del intermareal y las estaciones del año presentaron un efecto significativo, explicando un 61 y un 31\% de su variabilidad, respectivamente. Las zonas del intermareal mostraron ser la variable de mayor importancia a nivel de la composición del ensamble de algas, explicando el 53\% de la variación, mientras que la estacionalidad explicó solo el 18\% de ésta.

La determinación de 29 especies para la localidad de Maitencillo durante los 3 años de estudio, forman una pequeña parte $(\sim 23 \%)$ de las especies históricamente descritas para la Región de Valparaíso (e.g., Ramírez \& 
a)

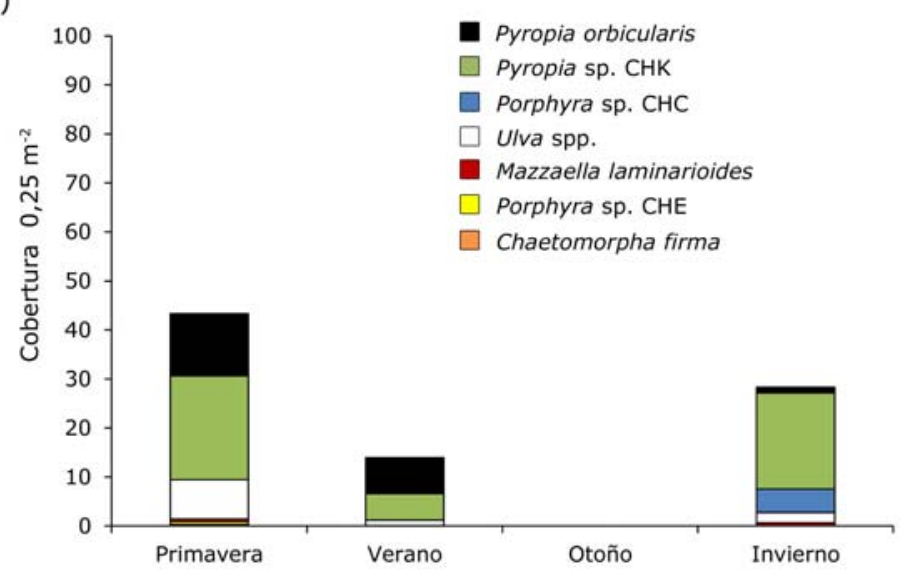

b)

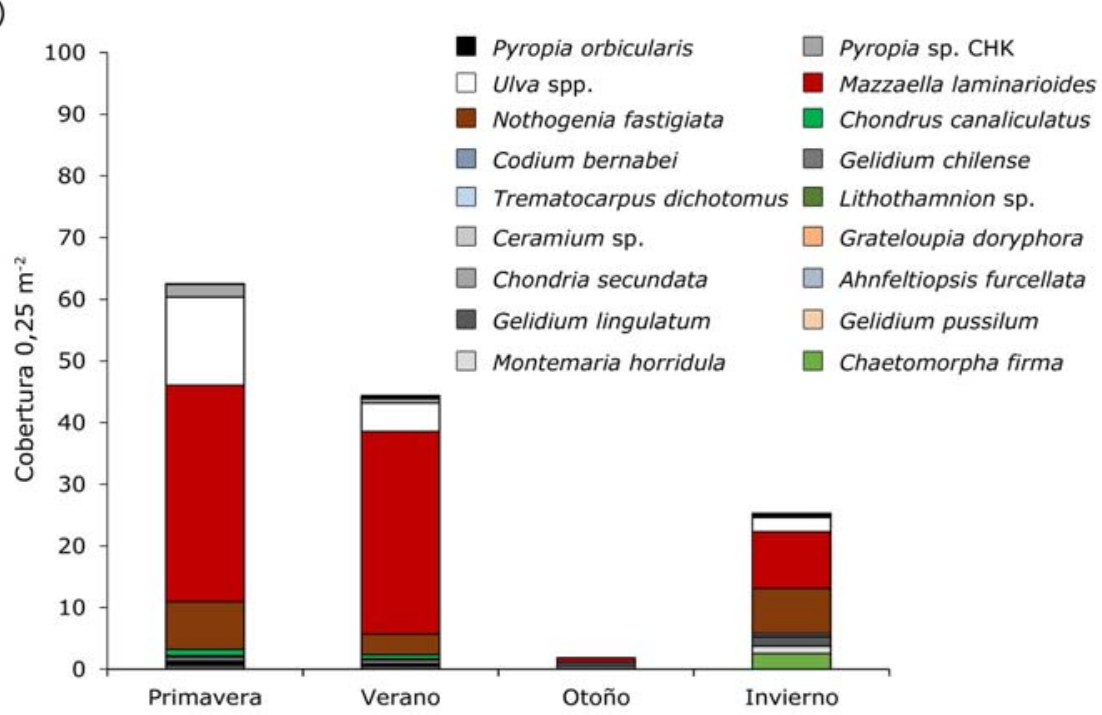

Figura 4. Cobertura de especies a) zona intermareal alta y b) zona intermareal media, durante los años 20132015 / Coverage of species a) high and b) the medium intertidal, during the years 2013-2015

Santelices 1991, Hoffman \& Santelices 1997). Esto indicaría diferencias entre localidades bajo estudio y épocas del año a lo largo de esta área geográfica. Sin embargo, es importante destacar, que actualmente la inclusión de la taxonomía molecular evidencia la necesidad de reescribir la distribución actual de la flora marina a lo largo de esta región. Por ejemplo, las especies de Porphyra referidas en estudios previos al 2014, no fueron registradas en este trabajo, lo cual se alinea con lo descrito por Ramírez et al. (2014) y Guillemin et al. (2016). De lo descrito por Guillemin et al. (2016) y por Ramírez et al. (2014), en este estudio fueron registradas las especies genéticas $P$. orbicularis, Pyropia sp. CHK, Porphyra sp. CHC y Porphyra sp. CHE.

La alta diversidad de este complejo en la zona de Maitencillo evidencia biotopos particulares con características micro-ambientales específicas, lo que permite el crecimiento y mantención de este complejo. Por otra parte, aunque no existen trabajos genéticos a nivel del complejo Ulva spp. en esta zona costera, nuestras observaciones durante el periodo de estudio evidencian una alta variación morfométrica de este complejo a lo largo de la zona intermareal, lo que sugiere también biotopos particulares de ocupación por especies particulares de este género.

Recientemente Zapata (2016) registró que, a lo largo de la zona intermareal de Maitencillo, existen rangos variables en términos de temperatura ambiental, humedad relativa y PAR (Radiación Fotosintéticamente Activa). Por ejemplo, en la zona alta y media del intermareal se registraron altas temperaturas entre los $40^{\circ} \mathrm{C}$ y $45^{\circ} \mathrm{C}$ 
a)

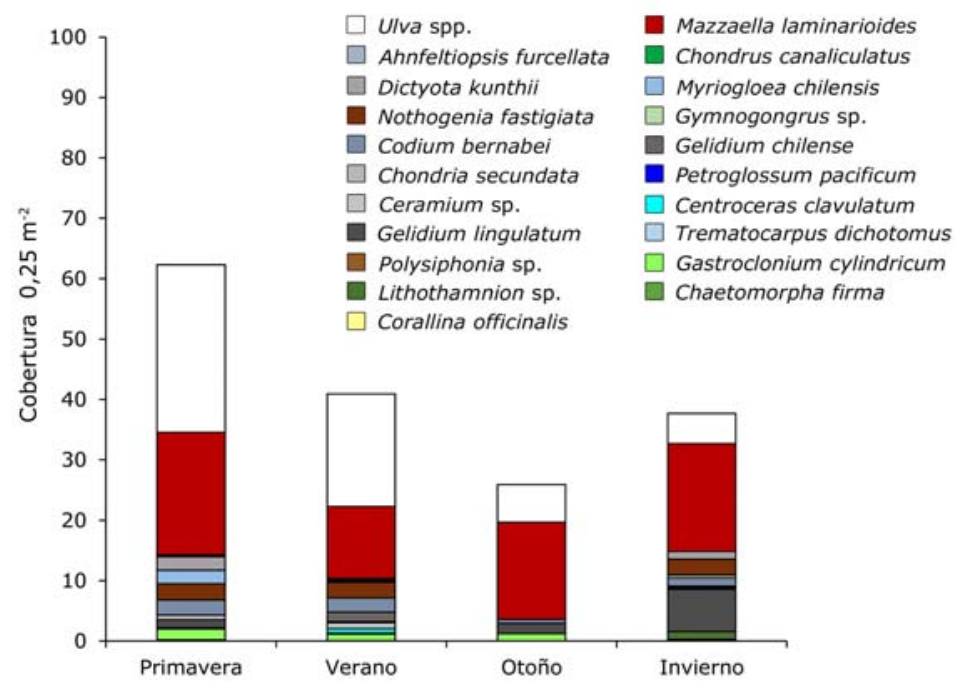

b)

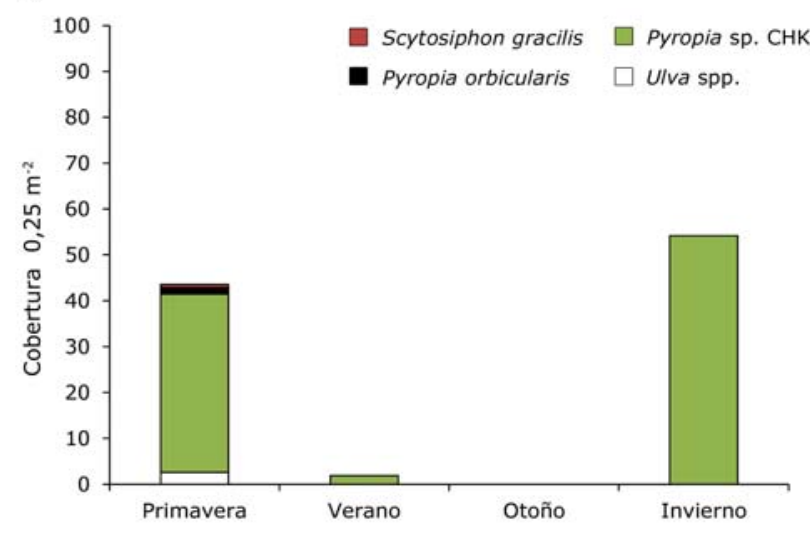

Figura 5. Cobertura de especies a) zona intermareal baja y b) paredones, durante los años 2013-2015 / Coverage of species a) low and b) step walls of the intertidal, during the years 2013-2015 durante verano, y solo de $25^{\circ} \mathrm{C}$ durante esta misma época en la zona de paredones. Por otra parte, en la zona baja del intermareal se registraron las menores temperaturas $\left(<15^{\circ} \mathrm{C}\right)$ durante un ciclo anual. Además, Zapata (2016) evidencia que estas diferencias micro-ambientales influyen en la capacidad de tolerar el estrés ambiental, explicando que aquellas especies de Pyropia que ocurren en la zona de paredones no son capaces de tolerar el mayor estrés que ocurre en la zona alta. Las especies que existen en este biotopo soportan altas horas de temperatura, baja humedad y alta intensidad lumínica principalmente durante las bajas de marea. Estas diferencias observadas por Zapata (2016) en especies de Pyropia pueden estar ocurriendo en otras especies (como en el caso del complejo Ulva spp.), lo que explicaría la variación de estas en términos de cobertura principalmente a lo largo de la zona intermareal.
Las especies que fueron registradas en la mayoría de las estaciones con alta cobertura, tales como Mazzaella laminarioides, Ulva spp., Codium bernabei, Gelidium lingulatum y Pyropia orbicularis, han sido descritas como especies con alto rango de tolerancia a factores de estrés ambiental, principalmente temperatura, PAR y desecación. Se ha descrito que en Ulva rigida C. Agardh, las altas temperaturas aumentan el asentamiento y la reproducción (Gao et al. 2017). Altas temperaturas en Ulva prolifera O.F. Müller, inducen cambios morfológicos externos y bioquímicos (actividad enzimática y contenido de proteínas) (Gao et al. 2016), explicando que esta plasticidad permite su mantención y crecimiento bajo altas temperaturas. En M. laminarioides se ha descrito que la coalescencia permite una mayor tolerancia a cambios de temperatura y salinidad (Medina et al. 2015). Además, presenta una alta tolerancia a PAR, donde la fotoinhibición 
podría explicar parte de su capacidad de tolerar ambientes sujetos a factores físicos estresantes (Navarro et al. 2016). En Gelidium lingulatum la alta tolerancia a amplios rangos de temperatura $\left(10-25^{\circ} \mathrm{C}\right)$ favorece su ocupación intermareal anual, reportándose mayores tasas de crecimiento a los $15^{\circ} \mathrm{C}$ (Oliger \& Santelices 1981). A diferencia de otras especies de Gelidium, que se ubican en zonas intermareales inferiores (e.g., Gelidium rex Santelices \& I.A.Abbott), presenta una mayor tasa de crecimiento a mayor dosis de fotones y días más largos (16 h) (Oliger \& Santelices 1981). Finalmente, en $P$. orbicularis su alta tolerancia a estrés ambiental como desecación, se explica por una plasticidad genética, lo que permite su amplia distribución geográfica y su ocurrencia en zonas altas del intermareal (e.g., ContrerasPorcia et al. 2011, 2012; Guillemin et al. 2016, Fierro et al. 2017, Latorre 2017).

Gelidium spp., Mazzaella laminarioides y las algas del complejo Porphyra / Pyropia representan recursos explotados económicamente (Buschmann et al. 2008). Justamente resultaron muy representativas en cobertura en la zona de estudio. Interesante son las variaciones estacionales y distribución de las especies pertenecientes a los géneros Porphyra y Pyropia, lo cual demuestra la existencia de adaptaciones particulares a la vida en el intermareal para un adecuado desarrollo de cada una de ellas. Esta información representa un insumo valioso para explorar métodos de propagación y cultivo para esas nuevas entidades genéticas, en donde el control abiótico de la reproducción y crecimiento, mediado por factores ambientales como temperatura, luz y fotoperiodo, ha sido fundamental en la domesticación de especies de Bangiales foliosas, en diferentes regiones del planeta (Katz et al. 2000, López-Vivas et al. 2015).

En este contexto, la información sobre las variaciones a lo largo de la zona intermareal y a nivel estacional, permiten reconocer actualmente el patrón de distribución vertical y estacional de diversas especies de macroalgas. Además, la descripción de la flora bentónica obtenida en este trabajo actualiza la diversidad de algas representativa de la Región de Valparaíso, Chile. Sin embargo, un análisis de mayor profundidad permitirá explicar las diferencias entre las especies que presentan una variabilidad significativa de ocurrencia espacio-temporal en esta zona costera.

\section{Agradecimientos}

Este trabajo fue financiado por FONDECYT 1120117, 1170881 y DI-501-14/R a LC-P.

\section{LITERATURA CITADA}

Alveal K. 1970. Estudios ficoecológicos en la región costera de Valparaíso. Revista de Biología Marina 14(1): 7-88.

Alveal K. 1971. El ambiente costero de Montemar y su expresión biológica. Revista de Biología Marina 14(3): 85119.

Arakaki N, K Alveal, ME Ramírez \& S Fredericq. 2011. The genus Callophyllis (Kallymeniaceae, Rhodophyta) from the central-south Chilean coast ( $33^{\circ}$ to $\left.41^{\circ} \mathrm{S}\right)$, with the description of two new species. Revista Chilena de Historia Natural 84(4): 481-499.

Benavides AG, JM Cancino \& FP Ojeda. 1994. Ontogenetic change in the diet of Aplodactylus punctatus (Pisces: Aplodactylidae): an ecophysiological explanation. Marine Biology 118(1): 1-5.

Buschmann AH, MDC Hernández-González \& D Varela. 2008. Seaweed future cultivation in Chile: perspectives and challenges. International Journal of Environment and Pollution 33(4): 432-456.

Calderon MS \& MS Boo. 2016. Phylogeny of Phyllophoraceae (Rhodophyta, Gigartinales) reveals Asterfilopsis gen. nov. from the southern hemisphere. Phycologia 55(5): 543-554.

Cancino J \& B Santelices. 1984. Importancia ecológica de los discos adhesivos de Lessonia nigrescens Bory (Phaeophyta) en Chile central. Revista Chilena de Historia Natural 57: 23-33.

Collantes G \& P Muñoz-Muga. 2009. Massive proliferation of Grateloupia intestinalis (Hooker fil. et Harvey) Setchell ex Parkinson (Rhodophyta, Halymeniaceae), a non-native species in Valparaíso Bay, central Chile. Revista de Biología Marina y Oceanografía 44(2): 527-532.

Contreras L, A Moenne \& JA Correa. 2005. Antioxidant responses in Scytosiphon lomentaria (Phaeophyceae) inhabiting copper-enriched coastal environments. Journal of Phycology 41(6): 1184-1195.

Contreras L, G Dennett, A Moenne, E Palma \& JA Correa. 2007. Molecular and morphologically distinct Scytosiphon species (Scytosiphonales, Phaeophyceae) display similar antioxidant capacities. Journal of Phycology 43: 1320-1328.

Contreras-Porcia L, D Thomas, V Flores \& JA Correa. 2011. Tolerance to oxidative stress induced by desiccation in Porphyra columbina (Bangiales, Rhodophyta). Journal of Experimental Botany 62: 1815-1829.

Contreras-Porcia L, S Callejas, D Thomas, C Sordet, G Pohnert, A Contreras, A La Fuente, MR Flores-Molina \& JA Correa. 2012. Seaweeds early development: detrimental effects of desiccation and attenuation by algal extracts. Planta 235: 337-348.

Fierro C, C López-Cristoffanini, A Meynard, C Lovazzano, F Castañeda, E Guajardo \& L Contreras-Porcia. 2017. Expression profile of desiccation tolerance factors in intertidal seaweed species during the tidal cycle. Planta 245(6): 1149-1164. 
Flores-Molina MR, D Thomas, C Lovazzano, A Núñez, J Zapata, M Kumar, JA Correa \& L Contreras-Porcia. 2014. Desiccation stress in intertidal seaweeds: Effects on morphology, antioxidant responses and photosynthetic performance. Aquatic Botany 113: 90-99.

Gao G, Z Zhong, X Zhou \& J Xu. 2016. Changes in morphological plasticity of Ulva prolifera under different environmental conditions: A laboratory experiment. Harmful Algae 59: 51-58.

Gao G, AS Clare, C Rose \& GS Caldwell. 2017. Eutrophication and warming-driven green tides (Ulva rigida) are predicted to increase under future climate change scenarios. Marine Pollution Bulletin 114: 439-447.

González AV \& B Santelices. 2017. Frequency of chimerism in populations of the kelp Lessonia spicata in central Chile. PloS ONE 12(2). e0169182. <doi:10.1371/journal.pone.0169182>

González AV, ME Chacana \& PC Silva. 2012. Codium bernabei sp. nov. (Bryopsidales, Chlorophyta), a coalescing green seaweed from the coast of Chile. Phycologia 51(6): 666-671.

González AV, J Beltrán \& B Santelices. 2014. Colonization and growth strategies in two Codium species (Bryopsidales, Chlorophyta) with different thallus forms. Phycologia 53(4): 353-358.

Guiler E. 1959. The intertidal ecology of Montemar Area, Chile. Papers and Proceedings of the Royal Society of Tasmania 93: 165-183.

Guillemin M-L, L Contreras-Porcia, ME Ramírez, EC Macaya, CB Contador, H Woods, C Wyatt \& J Brodie. 2016. The bladed Bangiales (Rhodophyta) of the South Eastern Pacific: molecular species delimitation reveals extensive diversity. Molecular Phylogenetics and Evolution 94: 814-826.

Guiry MD \& GM Guiry. 2018. AlgaeBase. World-wide electronic publication, National University of Ireland, Galway. <http://www.algaebase.org>

Hoffmann A \& B Santelices. 1997. Flora marina de Chile Central, 434 pp. Ediciones Universidad Católica de Chile, Santiago.

Hommersand M, G Leister, ME Ramírez, PW Gabrielson \& WA Nelson. 2010. A morphological and phylogenetic study of Glaphyrosiphon gen. nov. (Halymeniaceae, Rhodophyta) based on Grateloupia intestinalis with descriptions of two new species: Glaphyrosiphon lindaueri from New Zealand and Glaphyrosiphon chilensis from Chile. Phycologia 49(6): 554-573.

Jofré D, M Rivadeneira, F Tala \& M Thiel. 2014. Environmental tolerance of the two-invasive species Ciona intestinalis and Codium fragile: their invasion potential along a temperate coast. Biological Invasions 16: 2507-2527.

Katz S, Z Kizner, Z Dubinsky \& M Friedlander. 2000. Responses of Porphyra linearis (Rhodophyta) to environmental factors under controlled culture conditions. Journal of Applied Phycology 12(3): 535-542.
Latorre N. 2017. Análisis metabólico de factores de tolerancia al estrés oxidativo gatillado por desecación en la macroalga roja Pyropia orbicularis (Rhodophyta, Bangiales). Tesis de Magister en Biología Marina, Facultad de Ecología y Recursos Naturales, Universidad Andrés Bello, Santiago, 97 pp.

López-Vivas JM, R Riosmena-Rodríguez, AA JiménezGonzález, I Pacheco-Ruíz \& C Yarish. 2015. Growth and reproductive responses of the conchocelis phase of Pyropia hollenbergii (Bangiales, Rhodophyta) to light and temperature. Journal of Applied Phycology 27: 1561-1570.

Macaya EC \& GC Zuccarello. 2010. DNA barcoding and genetic divergence in the giant kelp Macrocystis (Laminariales). Journal of Phycology 46(4): 736-742.

Medina FJ, V Flores, AV González \& B Santelices. 2015. Coalescence increases abiotic stress tolerance in sporelings of Mazzaella laminarioides (Gigartinales, Rhodophyta). Journal of Applied Phycology 27(4): 1593-1598.

Meynard A, J Zapata, C Betancourtt, N Salas, C Espinoza, G Pérez, F Castañeda, ME Ramírez, C BulboaContador, M-L Guillemin \& L Contreras-Porcia. [In prep.]. Genetic and morphological differentiation of Porphyra and Pyropia species (Bangiales, Rhodophyta) coexisting in a rocky intertidal: habitat partitioning?

Montecinos A, BR Broitman, S Faugeron, PA Haye, F Tellier \& M-L Guillemin. 2012. Species replacement along a linear coastal habitat: phylogeography and speciation in the red alga Mazzaella laminarioides along the south east pacific. BMC Evolutionary Biology 12(1): 97-114.

Navarro NP, FL Figueroa, N Korbee, A Mansilla \& EM Plastino. 2016. Differential responses of tetrasporophytes and gametophytes of Mazzaella laminarioides (Gigartinales, Rhodophyta) under solar UV radiation. Journal of Phycology 52(3): 451-462.

O'hara RB \& DJ Kotze. 2010. Do not log-transform count data. Methods in Ecology and Evolution 1: 118-122.

Oliger P \& B Santelices. 1981. Physiological ecology studies on Chilean Gelidiales. Journal of Experimental Marine Biology and Ecology 53(1): 65-75.

Raffo MP, V Lo Russo \& E Schwindt. 2014. Introduced and native species on rocky shore macroalgal assemblages: zonation patterns, composition and diversity. Aquatic Botany 112: 57-65.

Ramírez ME. 2006. Algas marinas bentónicas. En: CONAMA (ed). Biodiversidad de Chile. Patrimonio y desafíos, pp. 352-361. Ocho Libros Editores, Santiago.

Ramírez ME \& C Osorio. 2000. Patrones de distribución de macroalgas y macro-invertebrados intermareales de la isla Robinson Crusoe, archipiélago de Juan Fernández, Chile. Investigaciones Marinas 28: 1-13.

Ramírez ME \& B Santelices. 1991. Catálogo de las algas marinas bentónicas de la costa temperada del Pacífico de Sudamérica, 437 pp. Facultad de Ciencias Biológicas, Pontificia Universidad Católica de Chile, Santiago. 
Ramírez ME, MR García-Huidobro \& N Orellana. 2008. Flora marina bentónica de caleta Quintay (Región de Valparaíso), litoral central de Chile. Boletín del Museo Nacional de Historia Natural, Chile 57: 9-19.

Ramírez ME, L Contreras-Porcia, M-L Guillemin, J Brodie, C Valdivia, MR Flores-Molina, A Nuñez, C Bulboa \& C Lovazzano. 2014. Pyropia orbicularis sp. nov. (Rhodophyta, Bangiaceae) based on a population previously known as Porphyra columbina from the central coast of Chile. Phytotaxa 158(2): 133-153.

Ramírez ML, C Bulboa, L Contreras-Porcia \& AM Mora. 2018. Flora marina bentónica de Quintay, 164 pp. Ril Editores, Santiago.

Rigby RA \& DM Stasinopoulos. 2005. Generalized additive models for location, scale and shape (with discussion). Applied Statistics 54(3): 507-554.

Santelices B \& ME Vera. 1984. Variación estacional de las floras marinas en la Caleta de Horcón. Chile Central. Phycologia Latinoamericana 2: 83-101.

Sordet C, L Contreras-Porcia, C Lovazzano, S Goulitquer, S Andrade, P Potin \& JA Correa. 2014. Physiological plasticity of Dictyota kunthii (Phaeophyceae) to copper excess. Aquatic Toxicology 150: 220-228.

Vásquez JA. 1992. Lessonia trabeculata, a subtidal bottom kelp in northern Chile: a case study for a structural and geographical comparison. In: Seeliger U (ed). Coastal plant communities of Latin America, pp. 77-89. Academic Press, New York.

Vásquez JA, S Zuñiga, F Tala, N Piaget, DC Rodríguez \& JA Vega. 2014. Economic valuation of kelp forests in northern Chile: values of goods and services of the ecosystem. Journal of Applied Phycology 26(2): 10811088.
Villaseñor-Parada C, A Pauchard \& E Macaya. 2014. Expansión del área de distribución de la macroalga introducida Schottera nicaeensis (Rhodophyta: Gigartinales) en la costa chilena: ¿evidencia de una invasión? Boletín de la Red Latinoamericana para el Estudio de Especies Invasoras 4(1): 19-27.

Villaseñor-Parada C, A Pauchard, ME Ramírez \& E Macaya. 2018. Macroalgas exóticas en la costa de Chile: patrones espaciales y temporales en el proceso de invasión. Latin American Journal of Aquatic Research 46(1): 147-165.

Wang Y, U Naumann, ST Wright \& DI Warton. 2012. Mvabund-an R package for model-based analysis of multivariate abundance data. Methods in Ecology and Evolution 3: 471-474.

Warton DI, ST Wright \& Y Wang. 2012. Distance-based multivariate analyses confound location and dispersion effects. Methods in Ecology and Evolution 3: 89-101.

Womersley HB. 1984. The marine benthic flora of Southern Australia. Part 1: 1-329. Govertment Printer, Adelaide.

Zapata J. 2016. Diferenciación ecológica entre ecotipos de dos especies hermanas de Pyropia (Bangiales, Rhodophyta) mediado por estrés ambiental. Tesis de Magister en Biología Marina, Facultad de Ecología y Recursos Naturales, Universidad Andrés Bello, Santiago, 127 pp.

Zardi GI, KR Nicastro, JF Costa, EA Serrão \& GA Pearson. 2013. Broad scale agreement between intertidal habitats and adaptive traits on a basis of contrasting population genetic structure. Estuarine, Coastal and Shelf Science 131: 140-148.

Zuur AF, EN Ieno, N Walker, AA Saveliev \& GM Smith. 2009. Mixed effects models and extensions in ecology with R, 574 pp. Springer, New York.

Recibido el 16 de mayo de 2017 y aceptado el 11 de abril de 2018

Editor Asociado: Pilar Muñoz M. 